\title{
Sensitivity of Harmonic Injection and its Spatial Evolution for Nonlinear Distortion Suppression in a TWT
}

\author{
A. Singh, J. E. Scharer (scharer@engr.wisc.edu), J. G. Wöhlbier and J. H. Booske \\ Room 513, 1500 Engineering Drive, University of Wisconsin, Madison, 53706 , \\ (608)263-1614, fax (608)265-2438
}

This paper presents an experimental investigation of the spatial evolution and sensitivity of third-order nonlinear distortion suppression obtained by harmonic injection in a Traveling Wave Tube to fundamental frequency shifts as well as injected secondharmonic amplitude and phase shifts. Harmonic injection schemes offer a promising solution to reduce the nonlinear distortion effects in a TWT [1-4]. The technique is readily implemented with simple circuits for generating the injected harmonic signal and adjusting its amplitude and phase. It is shown to yield high intermodulation suppression of up to $-30 \mathrm{~dB}$ (or $55 \mathrm{dBc}$ ). However, it is theoretically predicted to be quite sensitive to injected frequency, phase and amplitude variations.

Experiments were carried out on a custom-modified XWING (eXperimental WIsconsin Northrup-Grumman) TWT with two fundamental input tones of $\mathrm{f}_{\mathrm{a}}$ and $\mathrm{f}_{\mathrm{b}}$ at 1.90 and $1.95 \mathrm{GHz}$ at $15 \mathrm{dBm} /$ tone drive. Second-harmonics of these frequencies at $3.80\left(2 \mathrm{f}_{\mathrm{a}}\right)$ and $3.90\left(2 \mathrm{f}_{\mathrm{b}}\right) \mathrm{GHz}$ were then injected and iteratively adjusted in amplitude and phase to achieve maximum suppression of -31.4 and $-28.4 \mathrm{~dB}$ respectively for the upper $\left(2 \mathrm{f}_{\mathrm{b}}-\mathrm{f}_{\mathrm{a}}\right)$ and lower $\left(2 \mathrm{f}_{\mathrm{a}}-\mathrm{f}_{\mathrm{b}}\right)$ third-order intermodulation levels (3IMs) at the output.

Measurements of the wave spectrum at the sensors present along the tube axis reveal the destructive and constructive effects of the interfering modes generated by beating the fundamentals with the nonlinearly generated harmonic and the modes generated by beating the fundamentals with the injected harmonic. Since these modes have different growth rates and wavelengths $[4,6]$, the injected harmonic's amplitude and phase can be optimized to obtain suppression at a single axial location (chosen to be the output in this case). It has been shown in Ref. [4] that this location of maximum suppression can be shifted along the axis by changing the input parameters. Figure 1 shows the spectrum measurements at the input, sensor 1 (which is located almost halfway through the tube), sensor 4 (located close to the output port), and at the output.

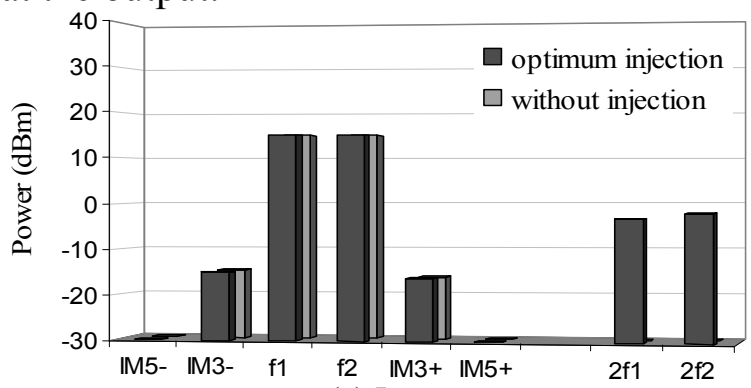

(a) Input

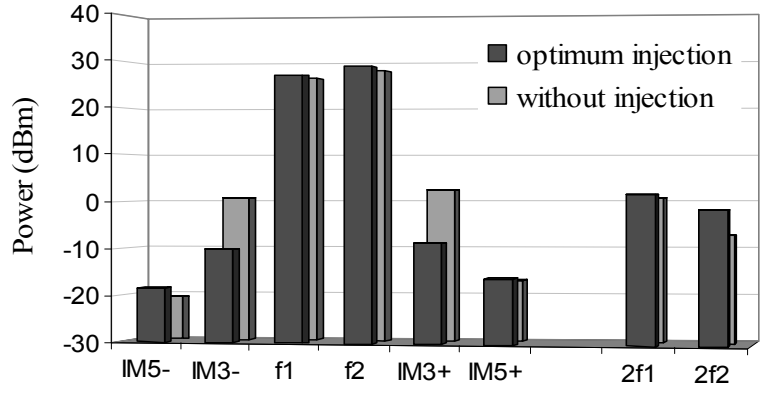

(b) Sensor 1

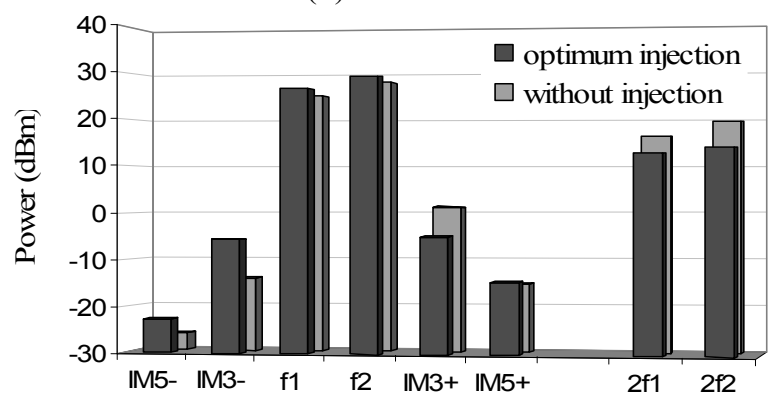

(c) Sensor 4 


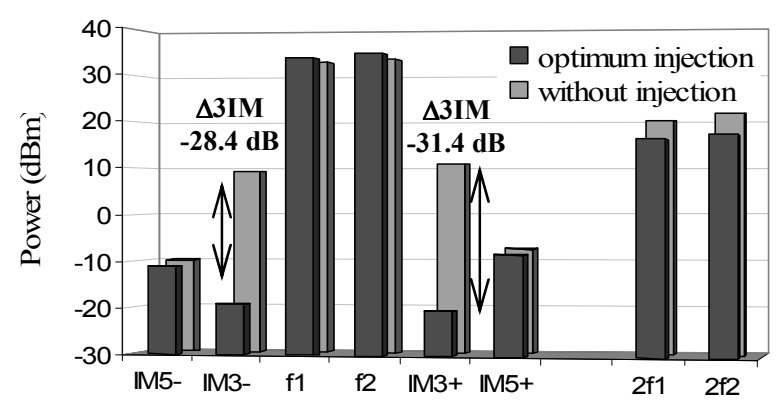

(d) Output

Fig. 1 Spatial evolution of the wave spectrum along the TWT axis with and without harmonic injection.

The input spectra shows that the optimum injected harmonic power required for maximum suppression are $-3.3 \mathrm{dBm}$ at 3.80 $\mathrm{GHz}$ and $-2.1 \mathrm{dBm}$ at $3.90 \mathrm{GHz}$. Thus, very small levels $(<17 \mathrm{dBc})$ of properly phased harmonics are needed to obtain 28.4-31.4 dB of 3IM suppression. The 3IM level at the input is less than $30 \mathrm{dBc}$ due to the preamplifiers. It can be seen that the output 3IM levels obtained with suppression are even lower than the initial level of 3IM present at the input. While all the frequencies show almost monotone growth along the axis without injection, the presence of the injected mode causes destructive and constructive effects along the axis. The results also reveal that suppressing the 3IMs results in -5 to -7 $\mathrm{dB}$ suppression of the output second harmonics as well. Thus, the injected amplitude and phase can be optimized to either achieve maximum suppression at a particular frequency or to obtain a desired suppression for several frequencies.

The significant difference in the power levels of the 3IMs between sensor 4 (which is located close to the output) and the output in Fig. 1 indicates that the suppression is quite sensitive to injected amplitude and phase. Measurements were carried out to investigate this sensitivity and the effect of changes in input fundamental frequency on the suppression achieved. For experimental simplicity, only one second harmonic at 3.90 $\mathrm{GHz}$ was injected to suppress the upper 3IM resulting in $-29.5 \mathrm{~dB}$ suppression. The injected second-harmonic phase and amplitude was then varied. Experimental results reveal that 3IM suppression of greater than $45 \mathrm{dBc}$ can be maintained for a $\pm 5^{\circ}$ phase and $\mathrm{a} \pm 1 \mathrm{~dB}$ amplitude variation in the injected harmonic. These tolerances can be readily achieved in practice. Further, it was observed that varying the frequency of the lower fundamental (keeping the upper fixed) from a $10 \mathrm{MHz}$ separation between the two fundamentals to $900 \mathrm{MHz}$ caused the suppression to increase by only $10 \mathrm{~dB}$ to a -19 $\mathrm{dB}(44 \mathrm{dBc})$ suppression level. These results show that second harmonic injection suppression is fairly insensitive (i.e. can be readily achieved in practice) to the input parameters.

Acknowledgements: This research was supported by a DUSD (S\&T) MultiUniversity Research Initiative (MURI) program on Innovative Microwave Vacuum Electronics managed by the United States Air Force Office of Scientific Research under Grant No. F49620-99-1-0297 and by Air Force Grant No. 49620-00-1-0088.

\section{References:}

[1] O. Sauseng, M.A. Huisjen, and W.E. Garrigus. IEDM Technical Digest, pages 411-414, 1975.

[2] W.E. Garrigus and M.L. Glick. Microwave Journal, 18:35-40, 1975.

[3] M.A. Wirth, A. Singh, J.E. Scharer, and J.H. Booske. IEEE Trans. Electron Devices, 49(6):1082--1084, 2002.

[4] J.G. Wöhlbier, J.H. Booske, and I. Dobson. To appear in IEEE Trans. Plasma Sci., 2004.

[5] D.J. Jennings, A. Bateman, and J.P. McGeehan. IEE Proc. Comm., 146(5): 297-302, 1999.

[6] A. Singh, J.G. Wöhlbier, J.H. Booske, and J.E. Scharer. Accepted with revisions in the Phys. Rev. Lett., 2004. 